Supplementary Information for

\title{
Algae biofilm reduces microbe-derived dissolved organic nitrogen discharges: performance and mechanisms
}

Yuan Lin, Liye Wang, Ke Xu, Hui Huang, Hongqiang Ren*

State Key Laboratory of Pollution Control and Resource Reuse, School of the Environment, Nanjing University, Nanjing 210023, Jiangsu, PR China

*Corresponding author: Professor Hongqiang Ren; Address: School of the Environment, Nanjing University, N.O.163, Xianlin Avenue, Nanjing 210023, Jiangsu, PR China; Email: hqren@nju.edu.cn; Phone: +86 2589680512

Pages: 24

Texts: 7

Tables: 2

Figures: 13 


\section{Supplementary Texts (Methods)}

\section{Text S1 Synthetic municipal wastewater composition}

The synthetic municipal wastewater contains $351.5 \mathrm{mg} / \mathrm{L} \mathrm{CH}{ }_{3} \mathrm{COONa}, 107.0 \mathrm{mg} / \mathrm{L}$ $\mathrm{NH}_{4} \mathrm{Cl}, 14.4 \mathrm{mg} / \mathrm{L} \mathrm{KNO}{ }_{3}, 1.54 \mathrm{mg} / \mathrm{L} \mathrm{KH}_{2} \mathrm{PO}_{4}, 2.94 \mathrm{mg} / \mathrm{L} \mathrm{CaCl} \cdot 2 \mathrm{H}_{2} \mathrm{O}$ and $7.35 \mathrm{mg} / \mathrm{L}$ $\mathrm{MgSO}_{4} \square 7 \mathrm{H}_{2} \mathrm{O}$ and $0.6 \mathrm{ml} / \mathrm{L}$ trace element solution to reach a composition of $240 \mathrm{mg} / \mathrm{L}$ chemical oxygen demand, $28 \mathrm{mg} / \mathrm{L} \mathrm{NH}_{4}{ }^{+}-\mathrm{N}, 2 \mathrm{mg} / \mathrm{L} \mathrm{NO}_{3}{ }^{-}-\mathrm{N}, 3.5 \mathrm{mg} / \mathrm{L} \mathrm{PO}_{4}{ }^{3-}-\mathrm{P}$. This configuration was chosen based on that of real municipal wastewater ${ }^{1}$. The trace element solution contains $1500 \mathrm{mg} / \mathrm{L} \quad \mathrm{FeCl}_{3} \square 6 \mathrm{H}_{2} \mathrm{O}, 180 \mathrm{mg} / \mathrm{L} \mathrm{KI}, 120 \mathrm{mg} / \mathrm{L}$ $\mathrm{ZnSO}_{4} \square 7 \mathrm{H}_{2} \mathrm{O}, 150 \mathrm{mg} / \mathrm{L} \mathrm{H}_{3} \mathrm{BO}_{3}, 120 \mathrm{mg} / \mathrm{L} \mathrm{MnCl}{ }_{2} \square 4 \mathrm{H}_{2} \mathrm{O}, 150 \mathrm{mg} / \mathrm{L} \mathrm{CoCl}{ }_{2} \square 6 \mathrm{H}_{2} \mathrm{O}$, $30 \mathrm{mg} / \mathrm{L} \mathrm{CuSO} \mathrm{Cu}_{4} \square 5 \mathrm{H}_{2} \mathrm{O}, 60 \mathrm{mg} / \mathrm{L} \mathrm{Na} \mathrm{MoO}_{4} \cdot 2 \mathrm{H}_{2} \mathrm{O} . \mathrm{NaHCO}_{3}$ was used to maintain the $\mathrm{pH}$ at $7.4 \pm 0.2$.

\section{Text S2 Solid phase extraction}

Waters Oasis HLB (6 cc, $150 \mathrm{mg}$ ), Waters Oasis WAX (6 cc, $150 \mathrm{mg})$ and Waters Oasis WCX (6 cc, $500 \mathrm{mg}$ ) SPE cartridges (Waters, USA) were used for DON recovery and desalination. WAX cartridge was pre-activated with $0.5 \% \mathrm{NH}_{4} \mathrm{OH}$ in methanol (MSgrade, Merck), methanol, and water (MS-grade, Merck); WCX cartridge was preactivated with $2 \%$ formic acid in methanol, methanol, and water; HLB cartridge was pre-activated with methanol and water. The three cartridges were connected in series to enrich DON. $500 \mathrm{~mL}$ for each sample was collected for extraction. DON eluted from WAX, WCX, and HLB with $10 \mathrm{~mL} 0.5 \% \mathrm{NH}_{4} \mathrm{OH}$ in methanol, $10 \mathrm{~mL} 2 \%$ formic acid in methanol, $10 \mathrm{~mL}$ methanol, respectively. Then, each eluent was concentrated to 1 $\mathrm{mL}$ using high purity nitrogen and stored at $-20^{\circ} \mathrm{C}$ in the dark before FTICR-MS analysis. 


\section{Text S3 Fourier-transform ion cyclotron resonance mass spectrometer (FTICR-}

\section{MS) analysis}

The extracted DON samples were diluted in 1:1 methanol: water to an identical DOC concentration for minimizing the matrix effect. Then, samples were analyzed with a 9.4T FT-ICR MS Bruker solariX (Bruker, Germany) equipped with negative-mode electrospray ionization under a needle voltage of $+3.5 \mathrm{kV}$. Samples were injected into the electrospray source by a syringe pump at a flow rate of $3.0 \mu \mathrm{L} / \mathrm{min}$, and 500 scans were collected for each one. The mass-to-charge ratio range of spectra was set at 100$800 \mathrm{~m} / \mathrm{z}$

\section{Text S4 Fourier-transform ion cyclotron resonance mass spectrometer (FTICR- MS) data processing}

This protocol follows the recommended order of operations provided by the package's author (https://github.com/skschum/MFAssignR\#recommended-order-of-operations). First, ${ }^{13} \mathrm{C}$ and ${ }^{34} \mathrm{~S}$ isotope masses were identified and filtered to avoid incorrect monoisotopic molecular formula assignments. Then a primary assignment of $\mathrm{C}, \mathrm{H}, \mathrm{O}$ using function MFAssignCHO was performed to assess the mass accuracy. The mass list was recalibrated again by choosing ten potential recalibrant series generated by RecalList function. Lastly, the ultimate molecular formulas were assigned using function MFAssign with the following elements: ${ }^{12} \mathrm{C}^{1} \mathrm{H}^{16} \mathrm{O}^{14} \mathrm{~N}_{0-12}{ }^{31} \mathrm{P}_{0-5}{ }^{32} \mathrm{~S}_{0-5}{ }^{35} \mathrm{Cl}_{0-2}$, and the mass accuracy was set to $1.0 \mathrm{ppm}$. All formulae should meet the following criteria 2: (1) $\mathrm{C} / 3 \leq \mathrm{H}+\mathrm{Cl} \leq 2 \mathrm{C}+\mathrm{N}+2 ;$ (2) $\mathrm{N} \leq \mathrm{O} ; \mathrm{S} \leq \mathrm{O}$. Double bond equivalents (DBE), nominal oxidation state of carbon (NOSC), modified aromaticity index $\left(\mathrm{AI}_{\text {mod }}\right)$, molecular lability index $\left(\mathrm{MLB}_{\mathrm{L}}\right), \mathrm{H} / \mathrm{C}$, and $\mathrm{O} / \mathrm{C}$ were calculated accordingly. ${ }^{3-5}$

\section{Text S5 Amplicon sequencing data processing}

Demultiplexed paired-end sequences $(\mathrm{n}=27)$ were denoised, and amplicon sequence variants (ASVs) were identified using DADA2 ${ }^{6}$. We pretrained a Naive Bayes 
classifier $^{7}$ against Greengenes database ${ }^{8}$ (v.8.15.13) and used it to assigned taxonomy for ASVs. The phylogenetic tree was constructed using SATé-enabled phylogenetic placement technique ${ }^{9}$ based on Greengenes $99 \%$ identity reference tree. All samples were rarefied to 15,000 sequences. Then the Faith's Phylogenetic Diversity (Faith's PD) ${ }^{10}$, Chao $1^{11}$, Shannon ${ }^{12}$, Simpson ${ }^{13}$ metrics, and weighted UniFrac distance ${ }^{14}$ for all samples were estimated. Analyses mentioned above were performed on QIIME2 ${ }^{15}$. The detailed definitions and explanations of Faith's PD, Chao1, Shannon, and Simpson metrics are as follow.

(1) Faith's PD is a measure of biodiversity which incorporates phylogenetic difference between species. Faith ${ }^{10}$ defined the phylogenetic diversity of a set of species as equal to the sum of the lengths of all those branches on the tree that span the set members (see the reference's Fig 1 to see how PD counts features).

(2) Chao1 index is an estimator of richness based on abundance, which can be calculated by the equation: $S_{e s t}=S_{o b s}+\frac{F^{2}}{2 G}$ where $S_{\text {est }}$ is the estimated number of species, $\mathrm{S}_{\mathrm{obs}}$ is the observed number of species in a sample, $\mathrm{F}$ is the number of singletons, and $\mathrm{G}$ is the number of doubletons.

(3) Shannon index (or entropy) estimates the diversity regarding the evenness. The more unequal the abundances of the species, the smaller the corresponding Shannon entropy. If practically all abundance is concentrated to one species, and the other species are scarce (even if there are many), the Shannon index approaches zero.

(4) Simpson index equals the probability that two entities taken at random from the dataset of interest (with replacement) represent the same type. Similar to Shannon entropy, it reflects the evenness of the community.

\section{Text S6 Reads quality control, assembly and binning}

The metagenomic analysis was conducted using our home-made pipeline (https://github.com/DOieGYuan/Easy-genomic-centric-metagenomics-pipepline). In brief, quality control was performed using FQC dashboard ${ }^{16}$ (v1.5.7) with parameters “ILLUMINACLIP:2:40:15:1:true SLIDINGWINDOW:4:15 MINLEN:50”. Qualified 
short reads from the same group were co-assembled into three scaffolds (i.e., $\mathrm{AB}_{1}, \mathrm{AB}_{2}$ and AS) using metaSPAdes ${ }^{17}$ (v3.14.0) with “-k 21,33,55,77,99”. Each co-assembly was binned into metagenome-assembled genomes (MAGs) using CONCOCT $^{18}$ (v1.1.0), MetaBAT2 ${ }^{19}$ (v2.2.15), MaxBin2 ${ }^{20}$ (v2.2.7), and then refined by metaWrap ${ }^{21}$ (v1.2.1). A total of 147 and 186 high-quality MAGs were recovered from AB and AS microbiome, respectively. Those MAGs accounted for $87.3 \%$ and $61.4 \%$ of sequences in $\mathrm{AB}$ and AS (Supplementary Data2), respectively. In addition, the comparison with 16S rRNA gene-based taxonomic profiles confirmed that the MAGs covered the most dominant taxa in corresponding systems (Supplementary Data2).

\section{Text S7 Estimating co-occurrence networks using CoNet}

Co-occurrence networks of ASVs-only ( $\mathrm{n}=9$ for each group) as well as ASVs and DON formulae ( $\mathrm{n}=9$ ) were constructed by $\mathrm{CoNet}^{22}$ (v2). Only ASVs with relative abundance $>0.1 \%$ and occurrence in over two thirds of the samples and DON with occurrence $>$ 5 were taken into consideration. Correlation-based methods "Pearson" and "Spearman", similarity-based method "Mutual Information", and distance-based methods "Bray Curtis" and "Kullback-Leibler" were used for the ensemble inference. Null distributions were calculated based on 200 randomized permutations, with "edgeScores" as routine and "shuffle_rows" as resampling strategy. $P$-values of an edge were merged into single $p$-value using "Brown's method" with 200 bootstraps replications and then the Benjamini and Hochberg false discovery rate (FDR) adjusted $p$-value was computed for multiple test correction. Edges with $\mathrm{FDR}<0.05$ were regarded as the robust interactions. 


\section{Supplementary Tables}

Table S1 Characteristics of stably present dissolved organic nitrogen (DON) in $\mathrm{AB}_{1}, \mathrm{AB}_{2}$ and $\mathrm{AS}$ reactors.

\begin{tabular}{ccccc}
\hline Group & Count & MW & AI $_{\text {mod }}$ & MLB $_{\mathrm{L}}$ \\
\hline $\mathrm{AB}_{1}$ & 311 & $301 \pm 57 \mathrm{~b}$ & $0.28 \pm 0.35 \mathrm{a}$ & $22.2 \%$ \\
$\mathrm{AB}_{2}$ & 185 & $314 \pm 51 \mathrm{a}$ & $0.28 \pm 0.49 \mathrm{a}$ & $20.5 \%$ \\
$\mathrm{AS}$ & 229 & $301 \pm 50 \mathrm{~b}$ & $0.29 \pm 1.22 \mathrm{a}$ & $49.8 \%$ \\
\hline $\mathrm{Group}$ & $\mathrm{DBE}$ & $\mathrm{DBEO}$ & $\mathrm{Cn}$ & $\mathrm{Nn}$ \\
\hline $\mathrm{AB}_{1}$ & $6.59 \pm 2.24 \mathrm{a}$ & $2.33 \pm 2.97 \mathrm{a}$ & $15.13 \pm 3.94 \mathrm{a}$ & $1.60 \pm 0.84 \mathrm{c}$ \\
$\mathrm{AB}$ & $6.96 \pm 2.66 \mathrm{a}$ & $2.76 \pm 3.11 \mathrm{a}$ & $14.78 \pm 4.13 \mathrm{~b}$ & $2.16 \pm 1.33 \mathrm{~b}$ \\
$\mathrm{AS}$ & $5.07 \pm 2.89 \mathrm{a}$ & $1.18 \pm 3.96 \mathrm{a}$ & $12.26 \pm 4.59 \mathrm{c}$ & $2.52 \pm 1.62 \mathrm{a}$ \\
\hline
\end{tabular}

MW, Molecular weight; $\mathrm{AI}_{\text {mod }}$, modified aromaticity index; $\mathrm{MLB}_{\mathrm{L}}$, molecular lability index; DBE, double bound; DBE, double bond equivalents; DBEO, oxygen-subtracted double bond equivalents; NOSC, nominal oxidation state of carbon; $\mathrm{Cn}$, the number of $\mathrm{C}$ atoms; $\mathrm{Nn}$, the number of $\mathrm{N}$ atoms. The letters behind the numbers indicate the significance of variances estimated from the Tukey HSD test at the confident level of adjusted $p$-value $<0.05$. 
Table S2 Putative phytochemical compounds (KEGG:br08003).

\begin{tabular}{ccccc}
\hline Formula & Compound & KEGG entry & Category & Occurrence \\
\hline $\mathrm{C}_{18} \mathrm{H}_{20} \mathrm{~N}_{2} \mathrm{O}_{6}$ & 3-Methoxytyramine-betaxanthin & $\mathrm{C} 17754$ & Lignin & $\mathrm{AB}_{1}, \mathrm{AB}_{2}, \mathrm{AS}^{*}$ \\
$\mathrm{C}_{17} \mathrm{H}_{27} \mathrm{NO}_{7}$ & Uplandicine & $\mathrm{C} 10412$ & Proteins/Amino sugars & $\mathrm{AB}_{1}, \mathrm{AB}_{2}{ }^{*}$ \\
$\mathrm{C}_{17} \mathrm{H}_{18} \mathrm{~N}_{2} \mathrm{O}_{6}$ & Miraxanthin-V & $\mathrm{C} 08557$ & Lignin & $\mathrm{AB}_{1}, \mathrm{AB}_{2}$ \\
$\mathrm{C}_{18} \mathrm{H}_{27} \mathrm{NO}_{5}$ & Nemorensine/Platyphylline/Sarracine & $\mathrm{C} 10353$ & Proteins/Amino sugars & $\mathrm{AB}_{1}, \mathrm{AB}_{2}{ }^{*}$ \\
$\mathrm{C}_{17} \mathrm{H}_{18} \mathrm{~N}_{2} \mathrm{O}_{5}$ & Miraxanthin-III & $\mathrm{C} 08556$ & Lignin & $\mathrm{AB}_{1}, \mathrm{AB}_{2}$ \\
$\mathrm{C}_{16} \mathrm{H}_{27} \mathrm{NO}_{6}$ & Europine & $\mathrm{C} 10301$ & Proteins/Amino sugars & $\mathrm{AB}_{1}, \mathrm{AB}_{2}{ }^{*}$ \\
$\mathrm{C}_{16} \mathrm{H}_{23} \mathrm{NO}_{6}$ & Monocrotaline & $\mathrm{C} 10350$ & Lignin & $\mathrm{AB}_{1}, \mathrm{AB}_{2}$ \\
$\mathrm{C}_{18} \mathrm{H}_{35} \mathrm{NO}_{3}$ & (+)-Prosopinine & $\mathrm{C} 10167$ & Lipids & $\mathrm{AB}_{1}, \mathrm{AB}_{2}, \mathrm{AS}$ \\
$\mathrm{C}_{16} \mathrm{H}_{27} \mathrm{NO}_{5}$ & Heliotrine & $\mathrm{C} 10324$ & Proteins/Amino sugars & $\mathrm{AB}_{1}, \mathrm{AB}_{2}, \mathrm{AS}^{*}$ \\
$\mathrm{C}_{16} \mathrm{H}_{23} \mathrm{NO}_{5}$ & Fulvine & $\mathrm{C} 10304$ & Lignin & $\mathrm{AB}_{1}, \mathrm{AB}_{2}$ \\
$\mathrm{C}_{14} \mathrm{H}_{16} \mathrm{~N}_{2} \mathrm{O}_{6}$ & Indicaxanthin & $\mathrm{C} 08549$ & Lignin & $\mathrm{AB}_{1}, \mathrm{AB}_{2}$ \\
$\mathrm{C}_{15} \mathrm{H}_{25} \mathrm{NO}_{5}$ & Indicine/Intermedine/Isolycopsamine/Lycopsamine/Rinderine & $\mathrm{C} 10326$ & Proteins/Amino sugars & $\mathrm{AB}_{1}, \mathrm{AB}_{2}, \mathrm{AS}^{*}$ \\
$\mathrm{C}_{18} \mathrm{H}_{35} \mathrm{NO}_{2}$ & Cassine & $\mathrm{C} 10136$ & Lipids & $\mathrm{AB}_{1}, \mathrm{AB}_{2}$ \\
$\mathrm{C}_{16} \mathrm{H}_{21} \mathrm{NO}_{4}$ & Convolvine/Phyllalbine & $\mathrm{C} 10856$ & Lignin & $\mathrm{AB}_{1}, \mathrm{AB}_{2}$ \\
\hline
\end{tabular}




\begin{tabular}{|c|c|c|c|c|}
\hline $\mathrm{C}_{15} \mathrm{H}_{25} \mathrm{NO}_{4}$ & Amabiline/Supinine & $\mathrm{C} 10263$ & Proteins/Amino sugars & $\mathrm{AB}_{1}, \mathrm{AB}_{2}, \mathrm{AS}$ \\
\hline $\mathrm{C}_{14} \mathrm{H}_{19} \mathrm{NO}_{5}$ & Harzianopyridone & $\mathrm{C} 10150$ & Lignin & $\mathrm{AB}_{1}, \mathrm{AB}_{2}^{*}, \mathrm{AS}^{*}$ \\
\hline $\mathrm{C}_{12} \mathrm{H}_{18} \mathrm{~N}_{2} \mathrm{O}_{5}$ & Hypoglycin B & $\mathrm{C} 08280$ & Proteins/Amino sugars & $\mathrm{AB}_{1}, \mathrm{AB}_{2}, \mathrm{AS}$ \\
\hline $\mathrm{C}_{13} \mathrm{H}_{21} \mathrm{NO}_{4}$ & Meteloidine & C10861 & Proteins/Amino sugars & $\mathrm{AB}_{1}, \mathrm{AB}_{2}, \mathrm{AS}^{*}$ \\
\hline $\mathrm{C}_{12} \mathrm{H}_{11} \mathrm{NO}_{4}$ & Casimiroin & $\mathrm{C} 10654$ & Lignin & $\mathrm{AB}_{1}, \mathrm{AB}_{2} *$ \\
\hline $\mathrm{C}_{11} \mathrm{H}_{11} \mathrm{NO}_{3}$ & Swietenidin B & $\mathrm{C} 10741$ & Lignin & $\mathrm{AB}_{1}, \mathrm{AB}_{2}, \mathrm{AS}^{*}$ \\
\hline $\mathrm{C}_{20} \mathrm{H}_{27} \mathrm{NO}_{3}$ & Hetisine & $\mathrm{C} 08687$ & Lignin & $\mathrm{AB}_{1}, \mathrm{AB}_{2}$ \\
\hline $\mathrm{C}_{20} \mathrm{H}_{27} \mathrm{NO}_{7}$ & Latifoline & C10344 & Lignin & $\mathrm{AB}_{1}, \mathrm{AB}_{2} *$ \\
\hline $\mathrm{C}_{19} \mathrm{H}_{27} \mathrm{NO}_{7}$ & Petasitenine & C10359 & Lignin & $\mathrm{AB}_{1}, \mathrm{AB}_{2}$ \\
\hline $\mathrm{C}_{19} \mathrm{H}_{27} \mathrm{NO}_{6}$ & Senkirkine & C10396 & Lignin & $\mathrm{AB}_{1}, \mathrm{AB}_{2} *$ \\
\hline $\mathrm{C}_{18} \mathrm{H}_{27} \mathrm{NO}_{6}$ & Rosmarinine & $\mathrm{C} 10380$ & Proteins/Amino sugars & $\mathrm{AB}_{1}, \mathrm{AB}_{2}$ \\
\hline $\mathrm{C}_{18} \mathrm{H}_{25} \mathrm{NO}_{6}$ & $\begin{array}{c}\text { Anacrotine/Angularine/Jacobine/Retrorsine/Usaramine/Senecion } \\
\text { ine N-oxide }\end{array}$ & C10277 & Lignin & $\mathrm{AB}_{1}, \mathrm{AB}_{2}{ }^{*}$ \\
\hline $\mathrm{C}_{18} \mathrm{H}_{25} \mathrm{NO}_{5}$ & Senecionine, Integerrimine, Senecivernine, Triangularine & $\mathrm{C} 06176$ & Lignin & $\mathrm{AB}_{1}, \mathrm{AB}_{2}$ \\
\hline $\mathrm{C}_{18} \mathrm{H}_{23} \mathrm{NO}_{5}$ & Seneciphylline & C10391 & Lignin & $\mathrm{AB}_{1}, \mathrm{AB}_{2} *$ \\
\hline $\mathrm{C}_{14} \mathrm{H}_{17} \mathrm{NO}_{6}$ & Prunasin; (R)-Prunasin & $\mathrm{C} 00844$ & Lignin & $\mathrm{AB}_{1}, \mathrm{AB}_{2} *$ \\
\hline $\mathrm{C}_{17} \mathrm{H}_{27} \mathrm{NO}_{3}$ & Nordihydrocapsaicin & C20216 & Lignin & $\mathrm{AB}_{1}, \mathrm{AB}_{2}, \mathrm{AS}$ \\
\hline
\end{tabular}




\begin{tabular}{lllll}
\hline $\mathrm{C}_{15} \mathrm{H}_{17} \mathrm{NO}_{4}$ & Dubinidine & $\mathrm{C} 10662$ & Lignin & $\mathrm{AB}_{1}, \mathrm{AB}_{2}$ \\
$\mathrm{C}_{19} \mathrm{H}_{24} \mathrm{~N}_{2} \mathrm{O}_{2}$ & Norajmaline & $\mathrm{C} 11810$ & Lignin & $\mathrm{AB}_{1}, \mathrm{AB}_{2}$ \\
$\mathrm{C}_{10} \mathrm{H}_{12} \mathrm{~N}_{4} \mathrm{O}_{6}$ & Xanthosine & $\mathrm{C} 01762$ & Lignin & $\mathrm{AB}_{1}, \mathrm{AB}_{2}, \mathrm{AS}$ \\
\hline
\end{tabular}

* Stochastically present 


\section{Supplementary Figures}

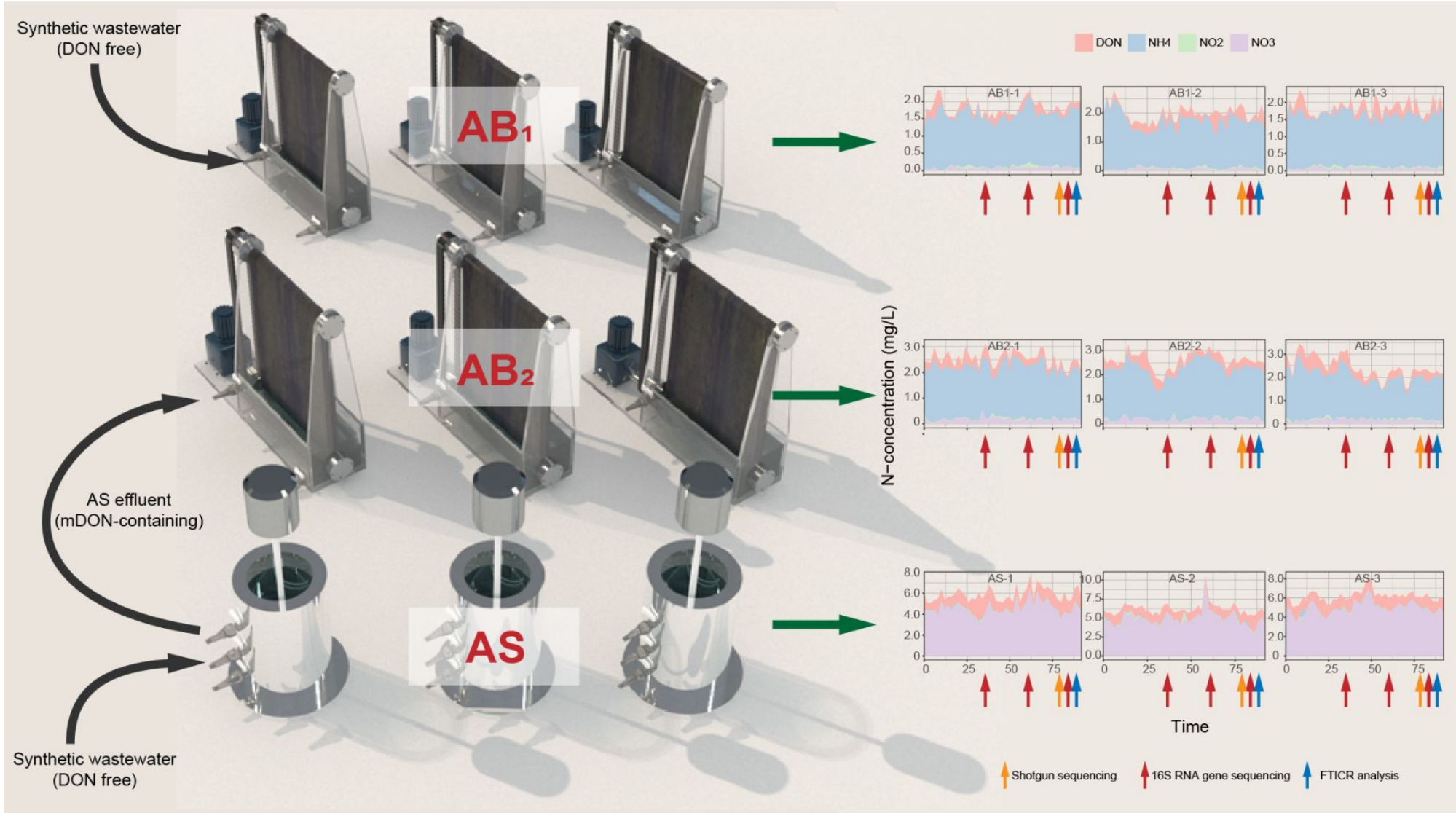

Figure S1 Schematic diagram of the experiment design and temporal patterns of the effluent nitrogen concentration in algae biofilm $(\mathrm{AB})$ reactors and activated sludge (AS) reactors. The predenitrification process (for AS reactors) consisted of a filling (15 $\mathrm{min})$, an anoxic (1.5 h), an aerobic (2.5 h), a settling (1.5 h), and a decanting $(15 \mathrm{~min})$ period. Other details of the operating parameters are illustrated in "Materials and Methods" in manuscript. 

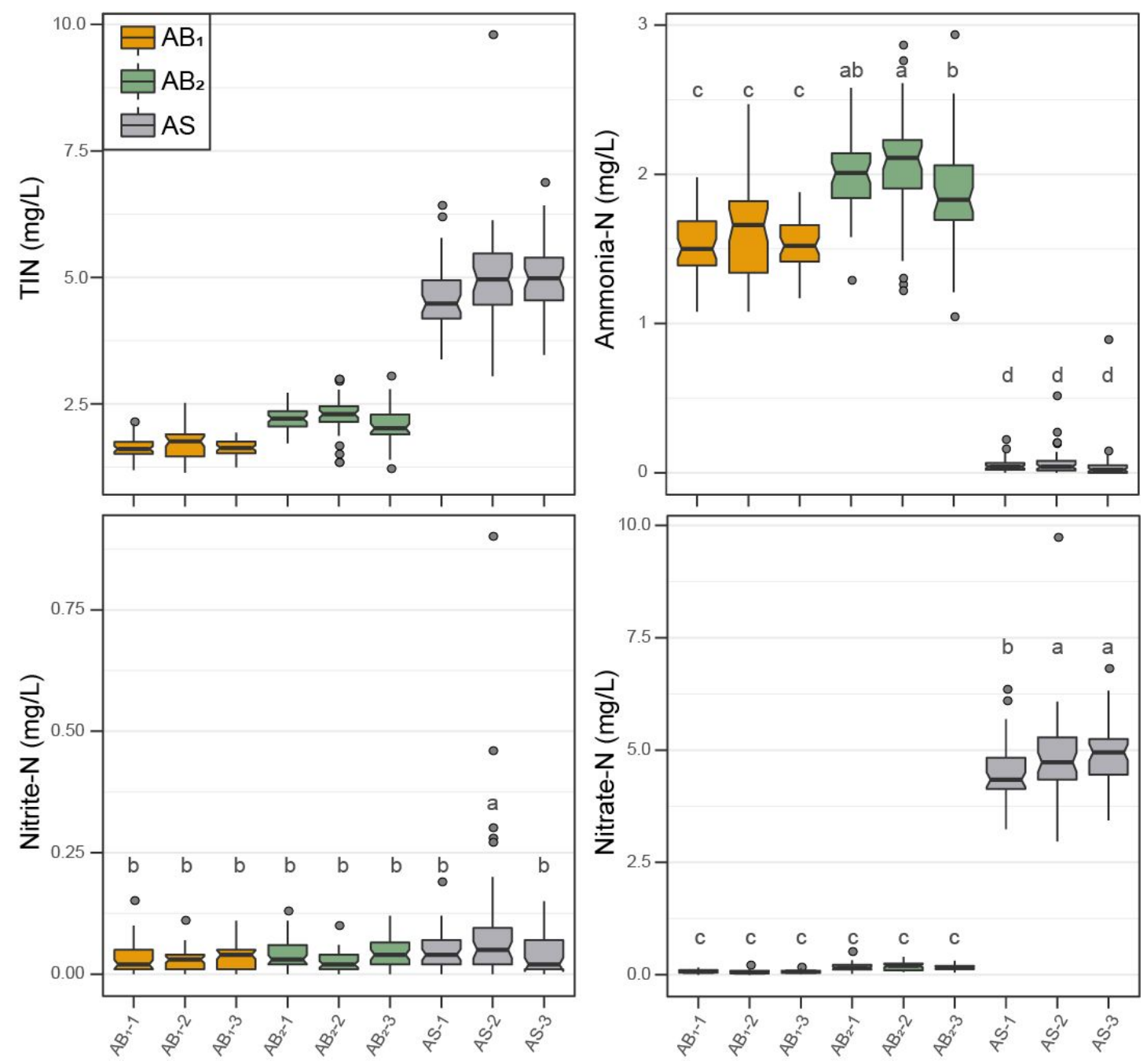

Figure S2 Concentrations of effluent dissolved inorganic nitrogen. The line in the middle indicates median and the notch gives a roughly $95 \%$ confidence interval of median. The lower and upper hinges correspond to the $25^{\text {th }}$ and $75^{\text {th }}$ quartiles. The whiskers extend from the hinges to the largest values no further than 1.5 -fold interquartile range from the hinges. Data beyond the end of the whiskers are outliers and are plotted individually as black dot. The letters above the boxplots indicate the significance of variances estimated from the Tukey HSD test at the confident level of adjusted $p$-value $<0.05$. The reactors with the same letter were not significantly different from each other concerning the mean value. 


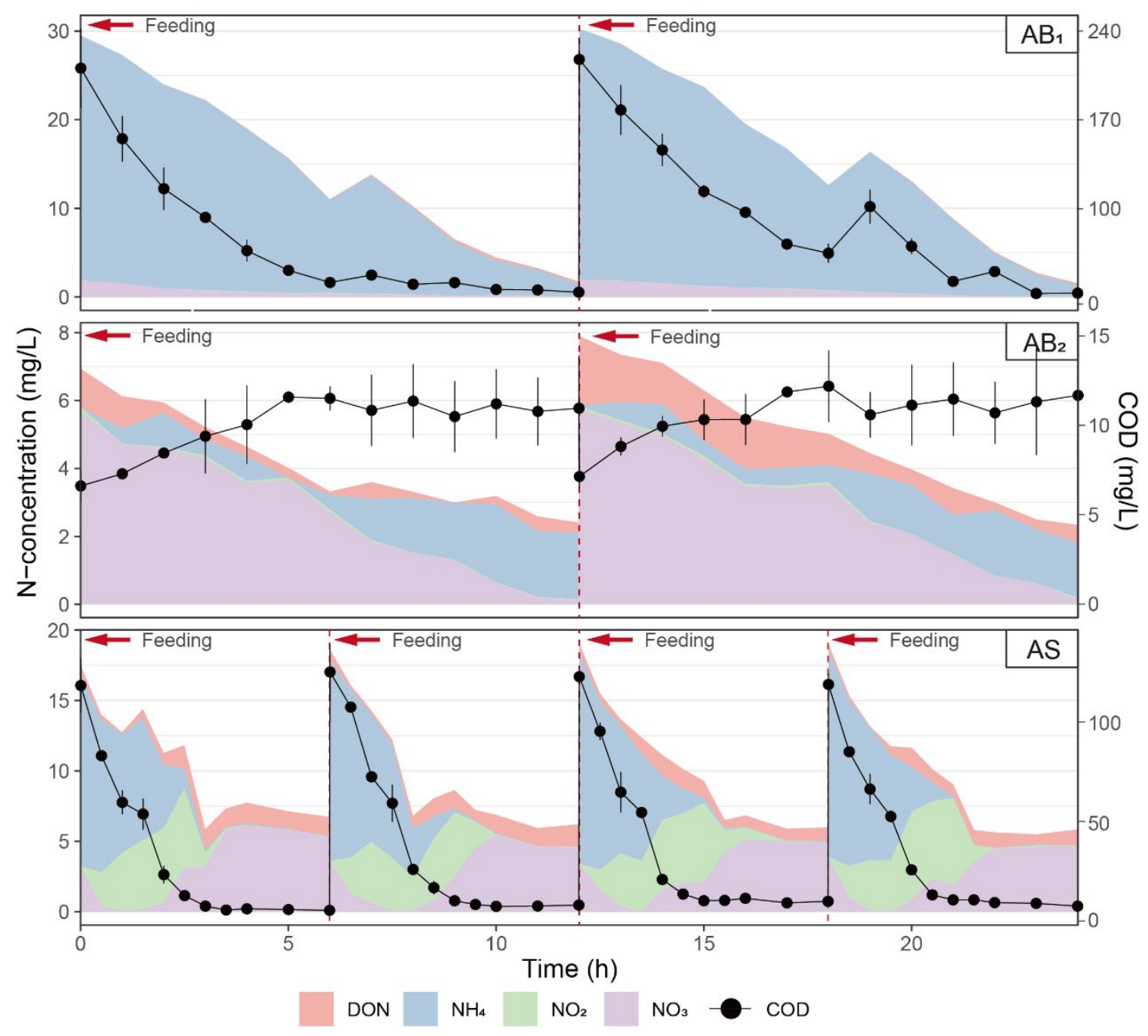

Figure S3 Chemical oxygen demand (COD) and nitrogen kinetics in typical cycles (in one day) of different groups. Stacked areas indicate the concentrations of different nitrogen species. Black dots refer to the COD concentrations. The error bar represents the standard deviation calculated from triplicates. 


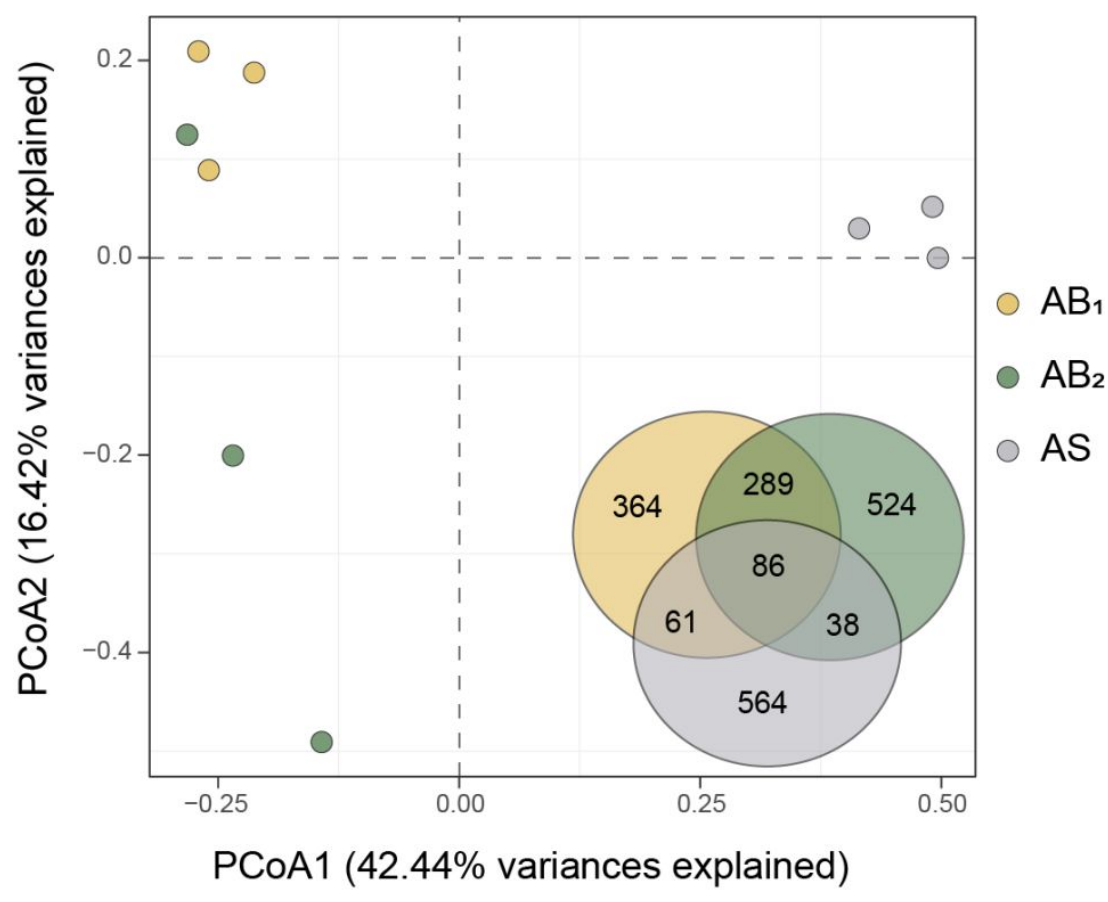

Figure S4 Principal component analysis (PCoA) of dissolved organic nitrogen (DON) compositions. The numbers in the Venn plot indicate the quantities of DON formulae in corresponding portions. 


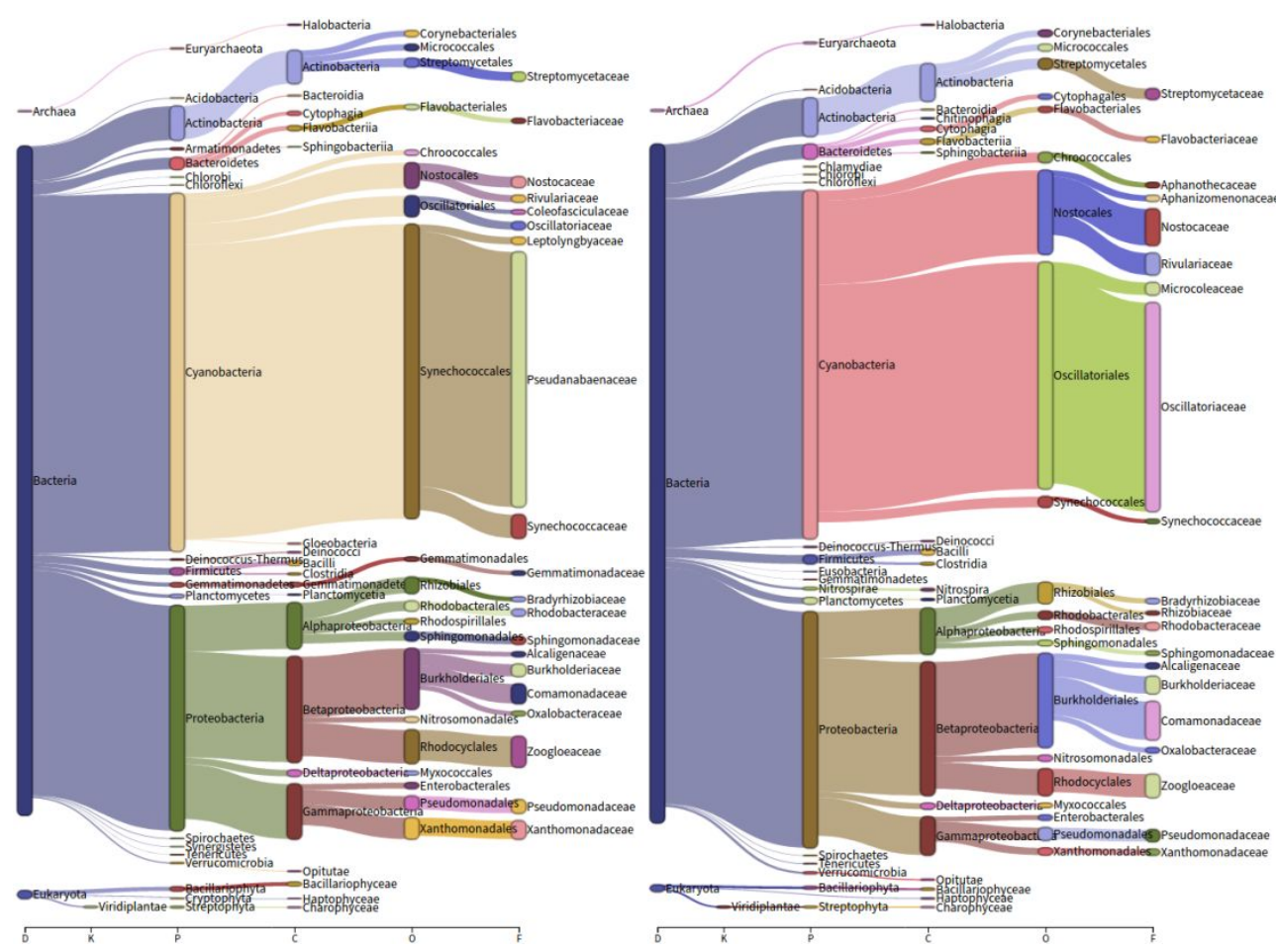

Figure S5 Short read-based taxonomic classification by Kraken2. Left panel is $\mathrm{AB}_{1}$ while right panel refers to $A B_{2}$. 
a

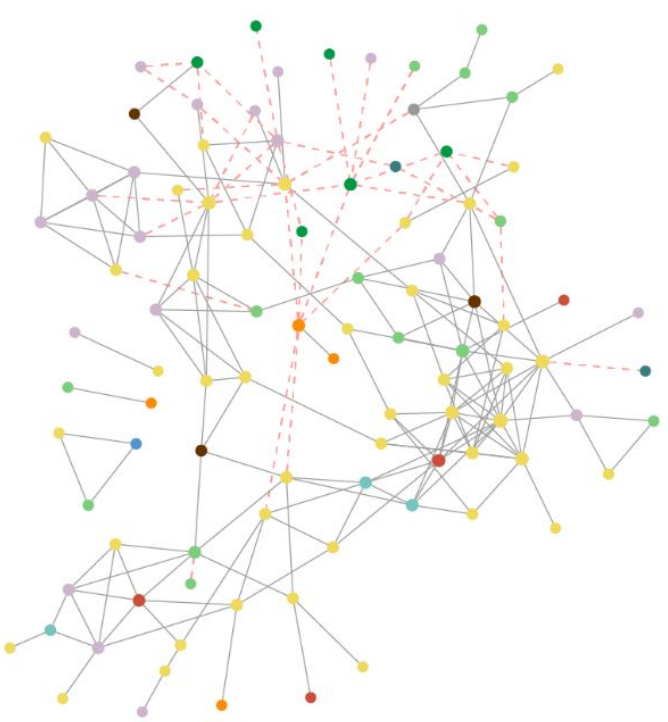

Network density: 0.040

Clustering coefficient: 0.274

Average degree: 3.837 b

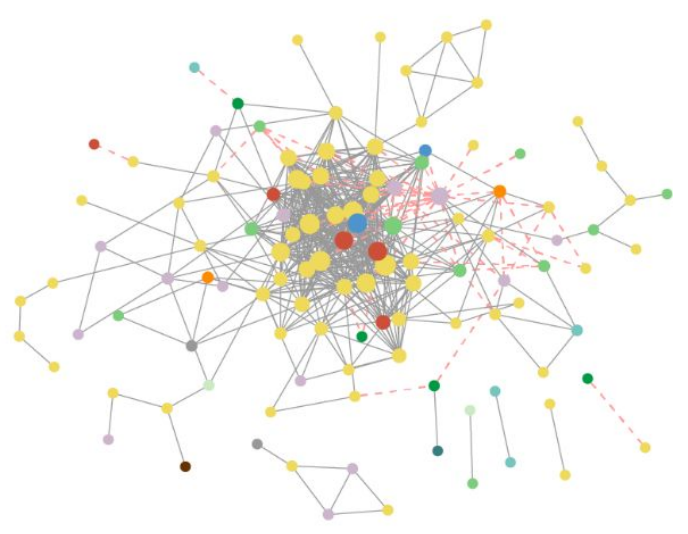

Network density: 0.081

Clustering coefficient: 0.431

Average degree: 9.235

C

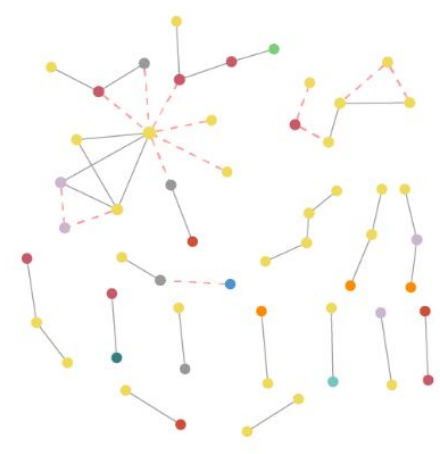

Network density: 0.031

Clustering coefficient: 0.128

Average degree: 1.630
Co-presence

Mutual exclusion

Acidobacteria

Actinobacteria

Bacteroidetes

Chlorobi

Chloroflexi

- Cyanobacteria

- Firmicutes

Nitrospirae

Planctomycetes

Proteobacteria

Verrucomicrobia

Figure S6 Co-occurrence networks of amplicon sequence variants (ASVs). (a)

$\mathrm{AB}_{1} .(\mathbf{b}) \mathrm{AB}_{2} .(\mathbf{c}) \mathrm{AS}$. 


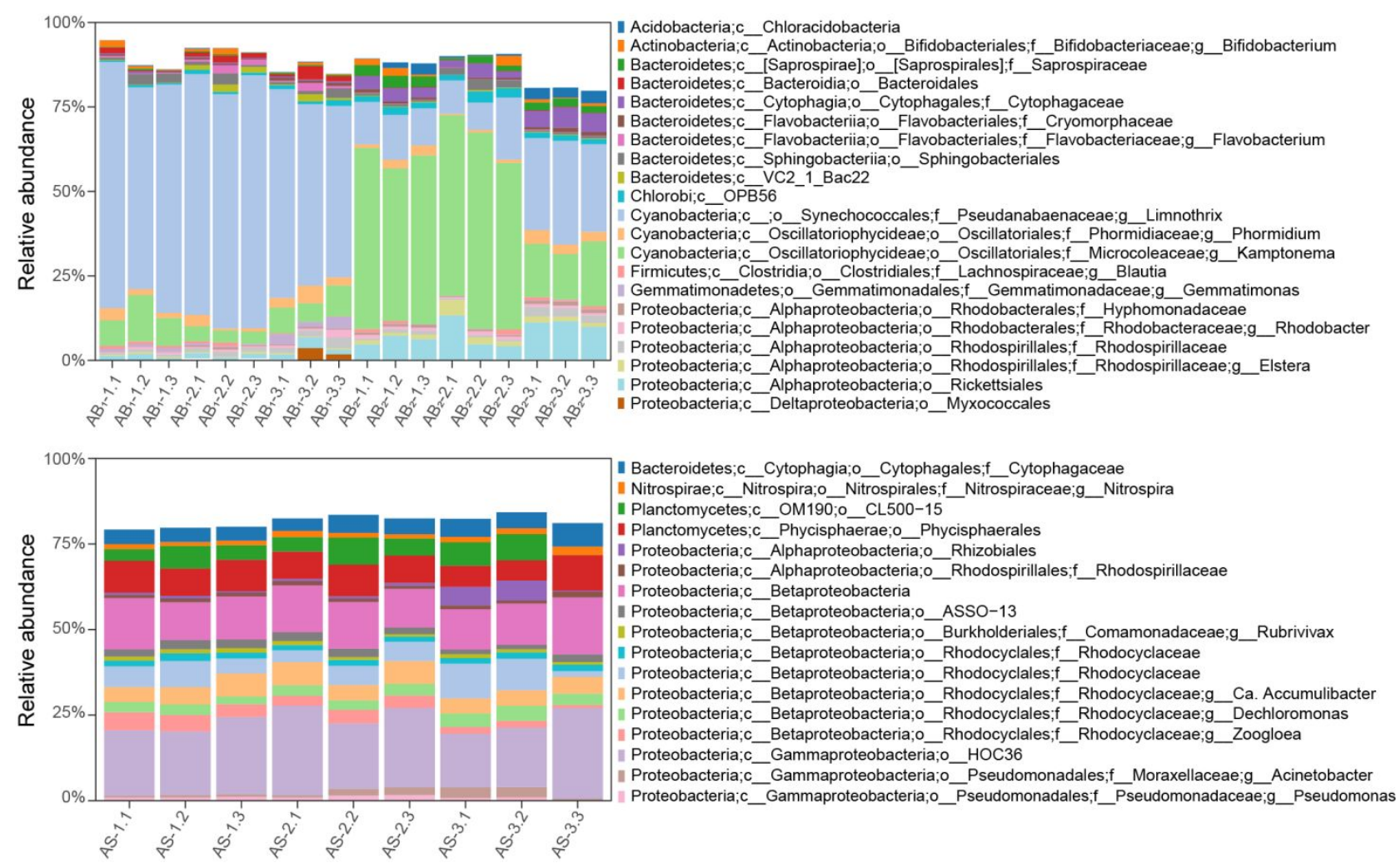

Figure S7 Taxonomic classification of algae biofilm (AB, above) microbiotas and activated sludge (AS, below) microbiota at genus level. 
a

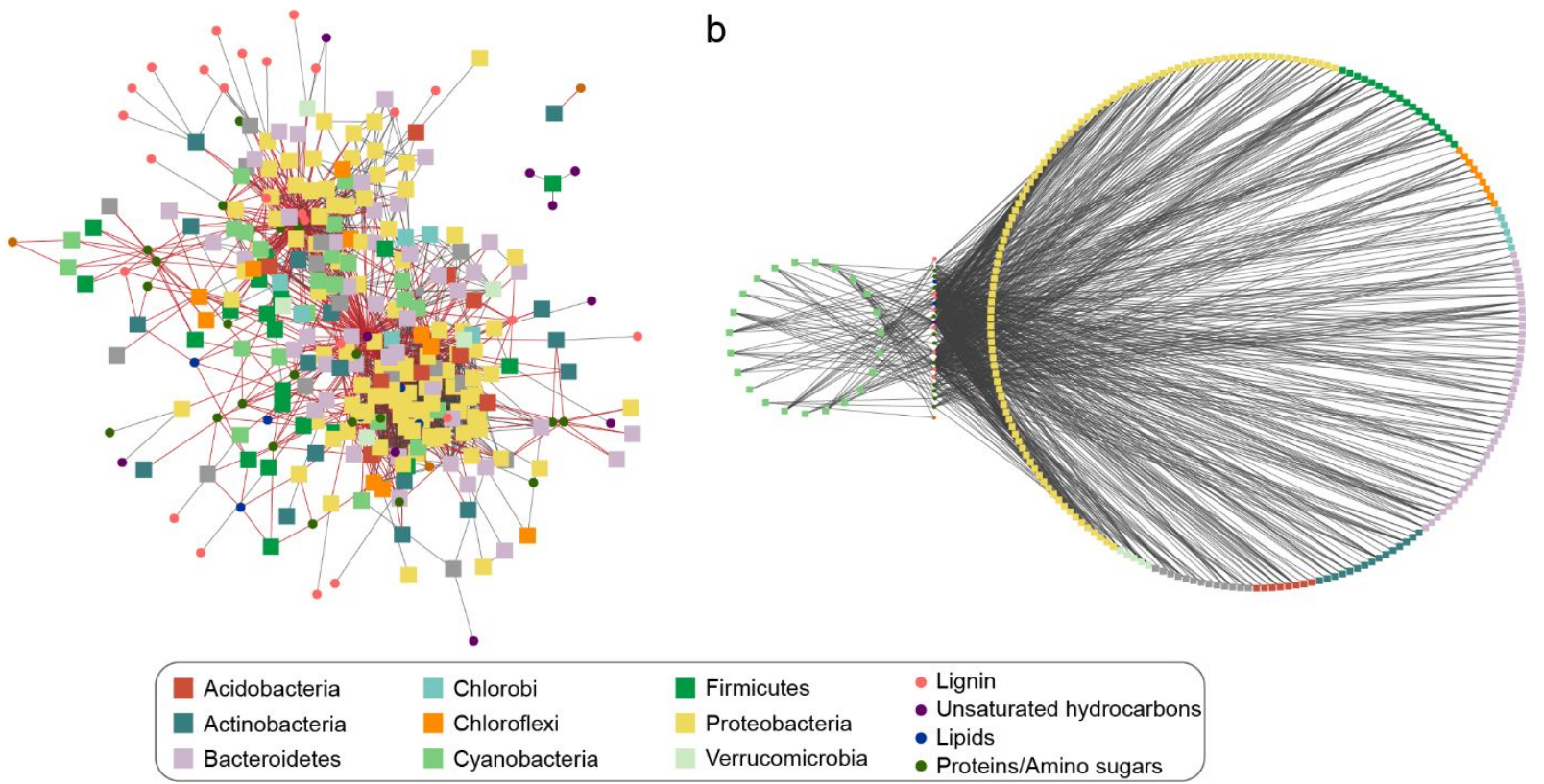

Figure S8 Co-occurrence network of amplicon sequence variants (ASVs) and

dissolved organic nitrogen (DON). (a) Co-occurrence network of ASVs and DON

formulae. Black lines indicate co-present interactions and red lines refer to mutual-

exclusion associations. (b) 727 edges linked cyanobacteria with other bacteria via

certain DON compounds. 


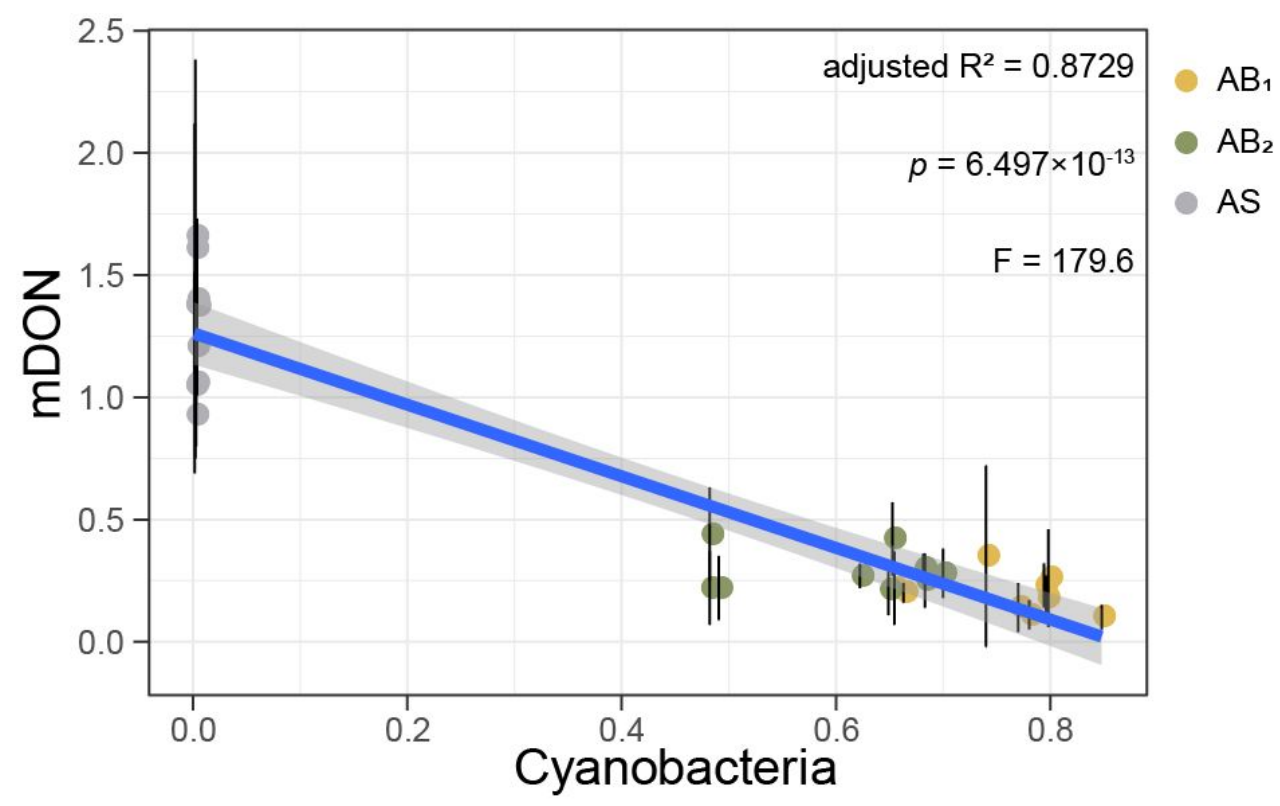

Figure S9 Linear regression model of the cyanobacteria abundance and dissolved organic nitrogen (DON) concentration. Error bars indicate the standard deviations of DON concentrations from three biological replicates. 
a
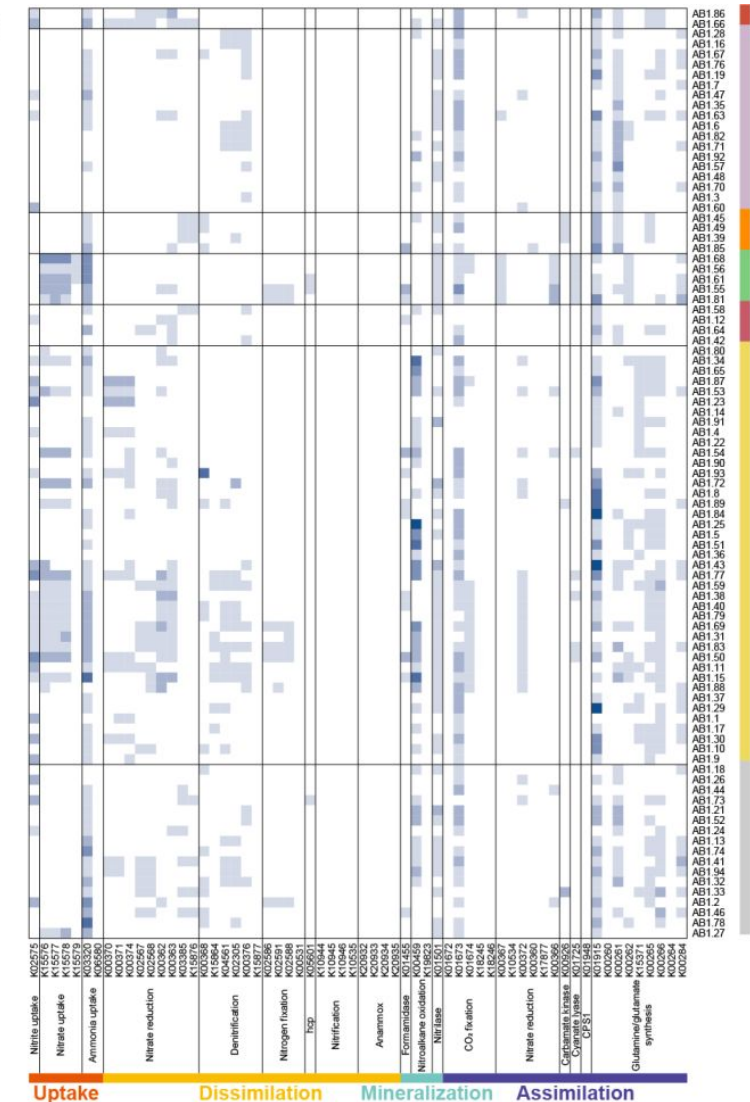

b

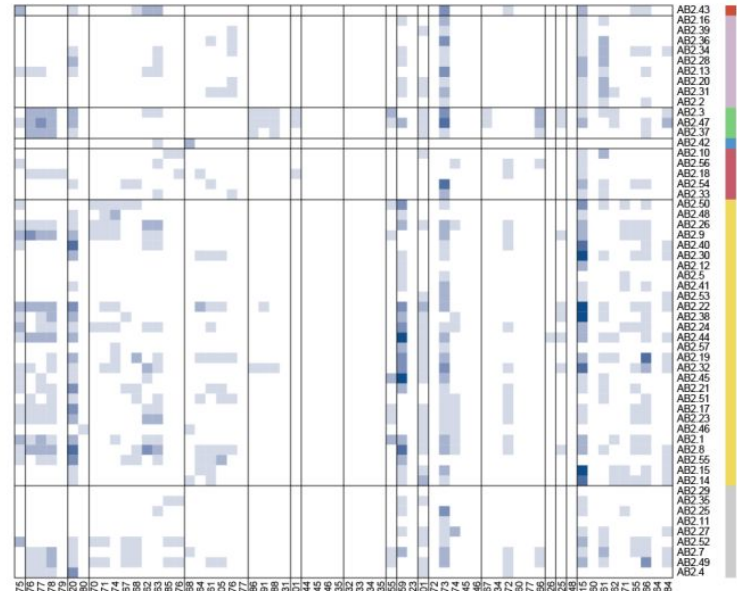

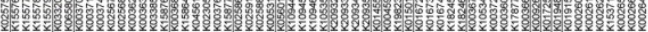

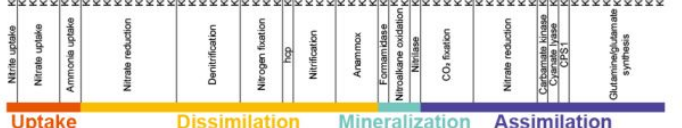

$\begin{array}{llll}\text { Acidobacteria } & \text { Cyanobacteria } & \text { Proteobacteria } & \begin{array}{c}\text { Gene copies } \\ \text { per genome }\end{array} \\ \text { Bacteroidetes } & \text { Nitrospirae } & \text { Others } & -v_{4}^{>6} \\ \text { Chloroflexi } & \text { Planctomycetes } & & \square_{0}^{2}\end{array}$
C

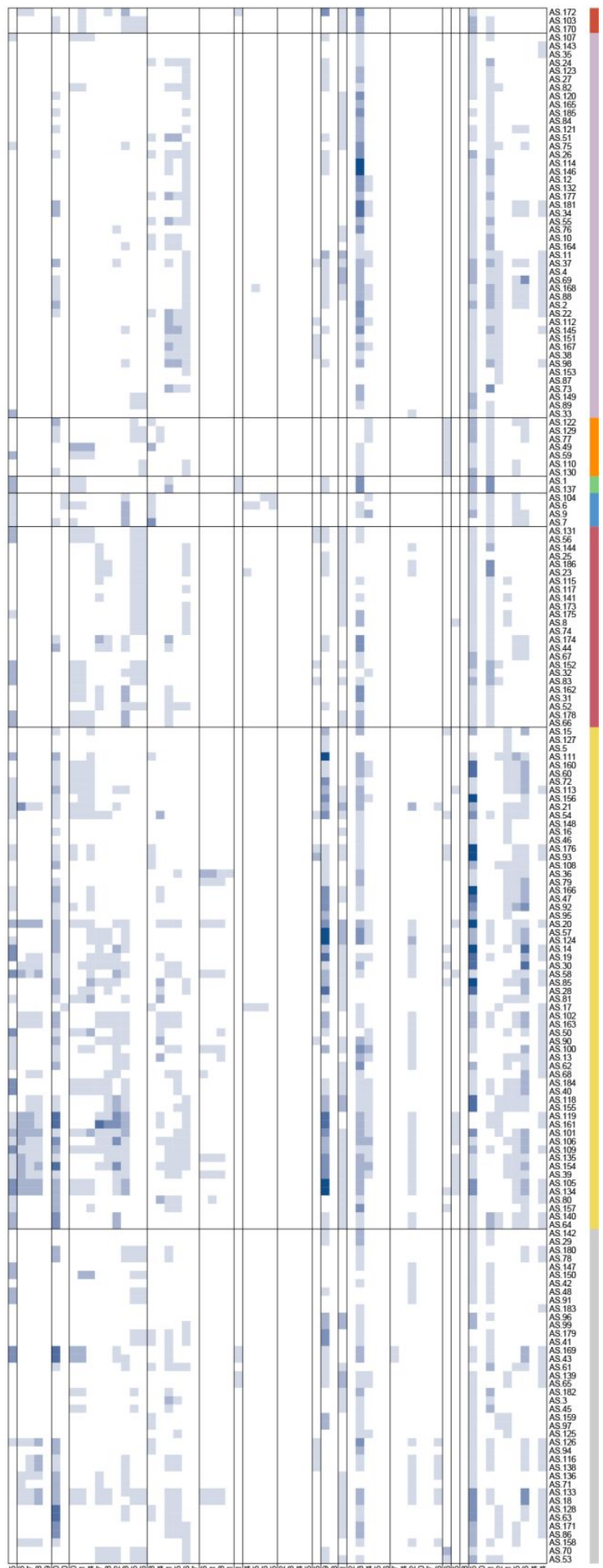

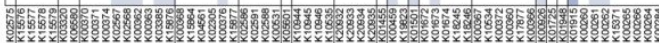

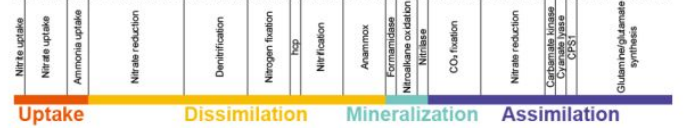

Figure S10 Presence of genes involving in nitrogen uptake, dissimilation,

mineralization and assimilation in metagenomic-assembled genomes (MAGs). (a)

$$
\mathrm{AB}_{1} \text {. (b) } \mathrm{AB}_{2} .(\mathbf{c}) \mathrm{AS} .
$$




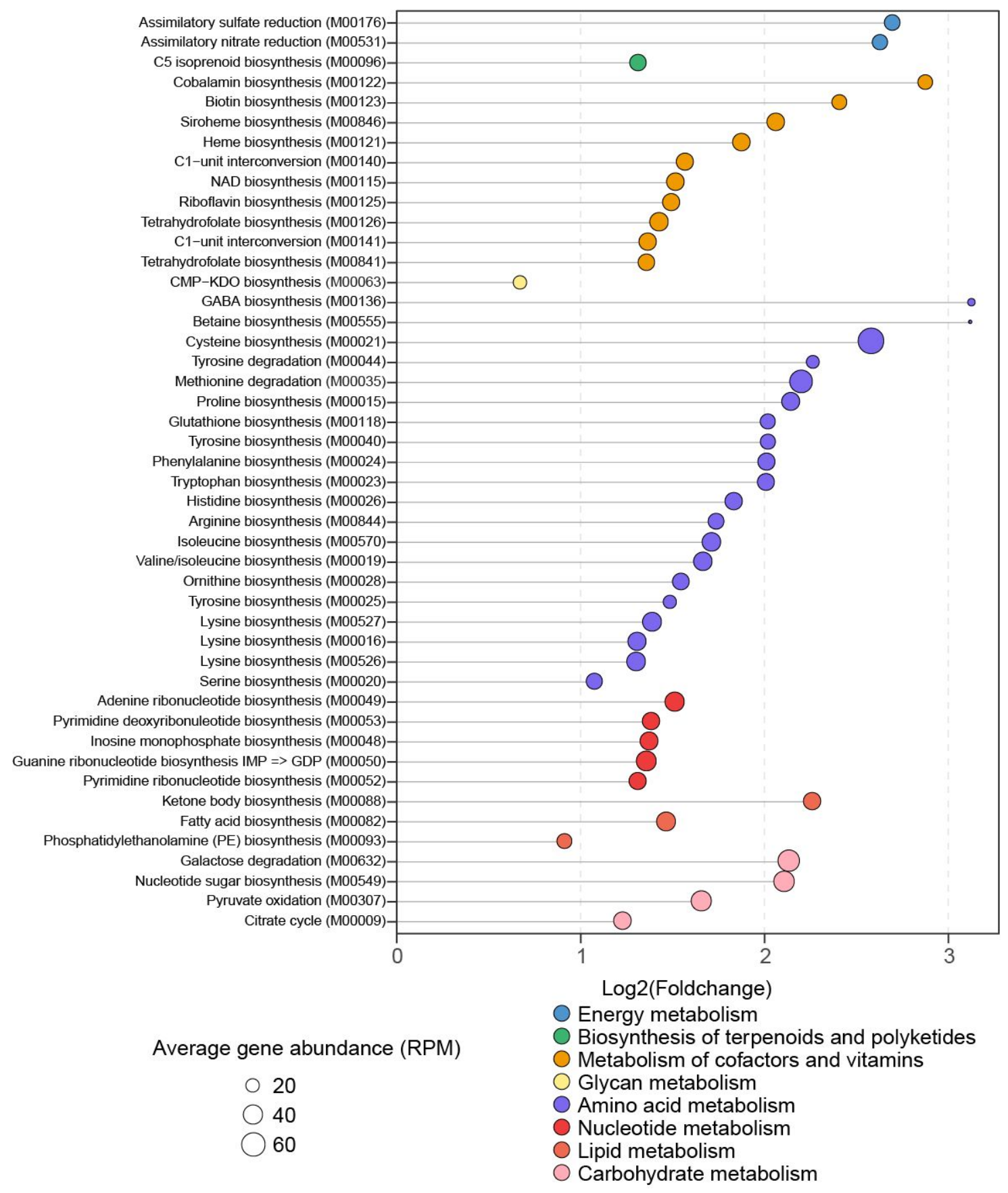

Figure S11 Similarity percentage (SIMPER) analysis of the KEGG Modules that drove the functional differences between $\mathrm{AB}_{2}$ microbiome and $\mathrm{AS}$ microbiome. 


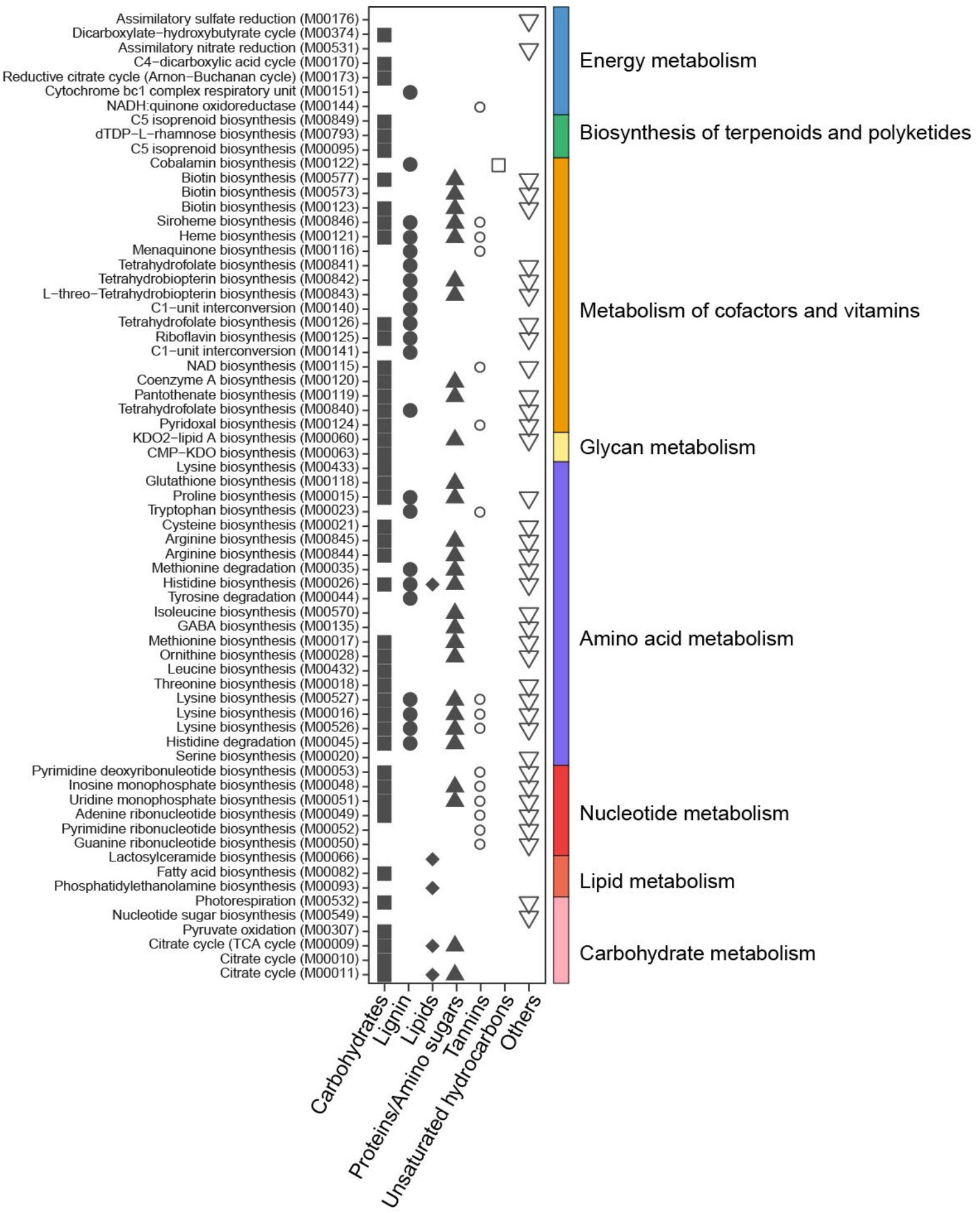

Figure S12 Categories of the nitrogenous metabolites within the significantly

different KEGG Modules. 
a

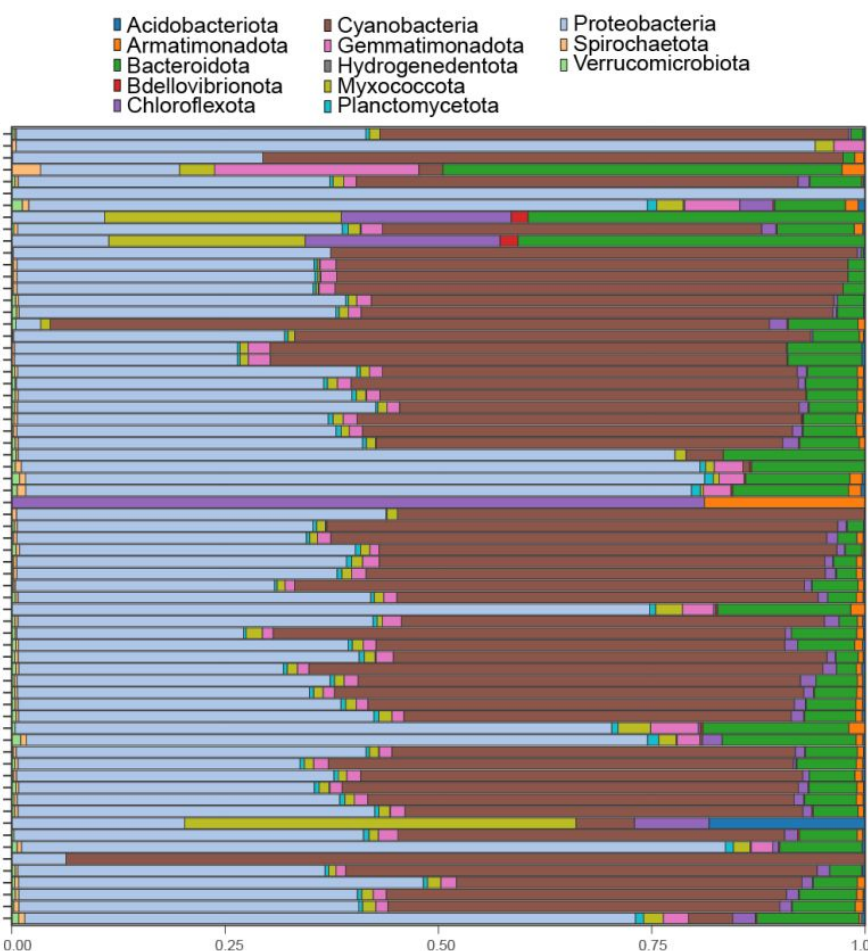

C

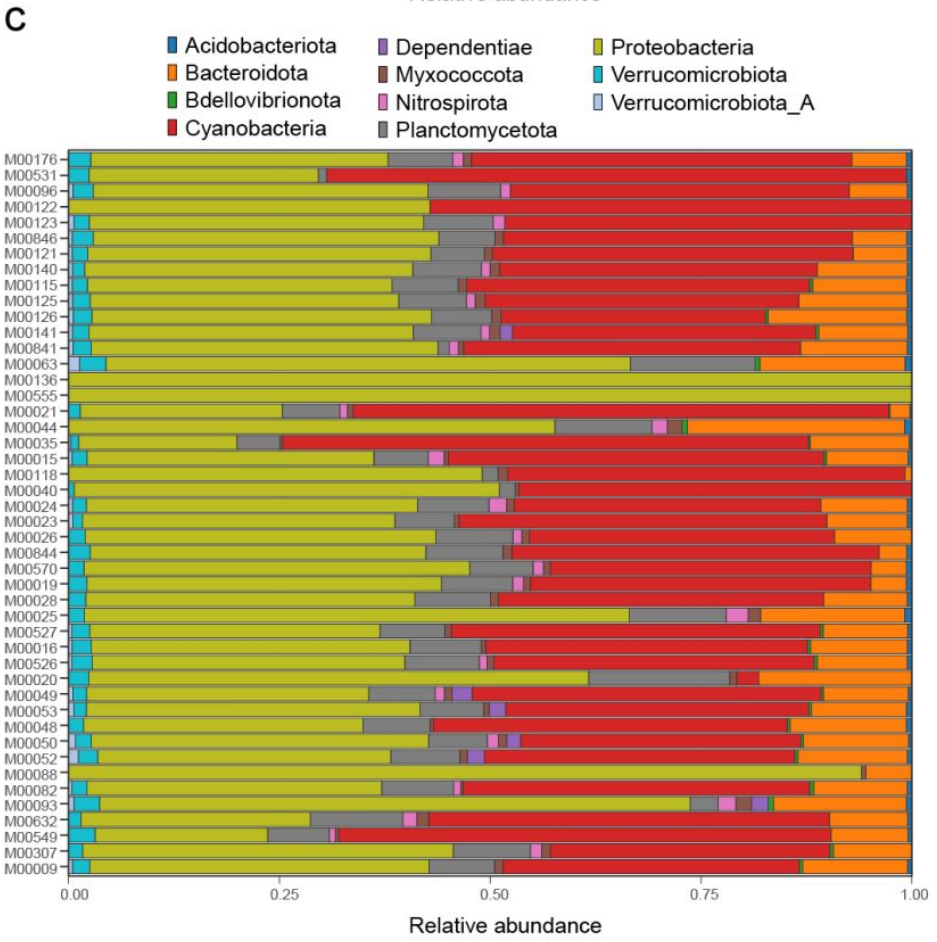

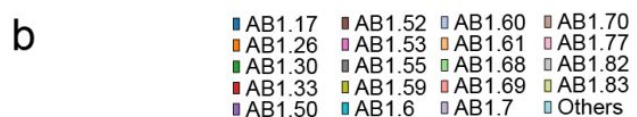

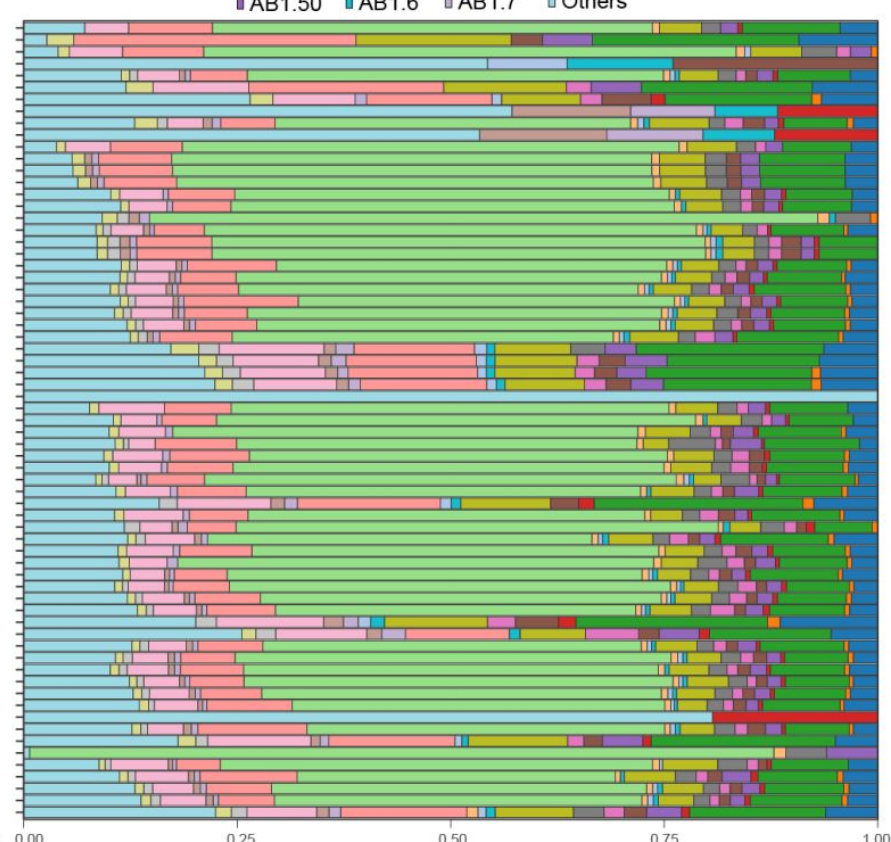

d Relative abundance

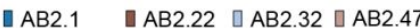

AB2.14 $\triangle A B 2.24 \square A B 2.34 \square A B 2.49$

AB2.15 AB2.29 AB2.36 AB2.54

- $\mathrm{AB} 2.17 \square \mathrm{AB} 2.3 \quad \square \mathrm{AB} 2.42 \square \mathrm{AB} 2.8$

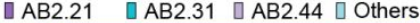

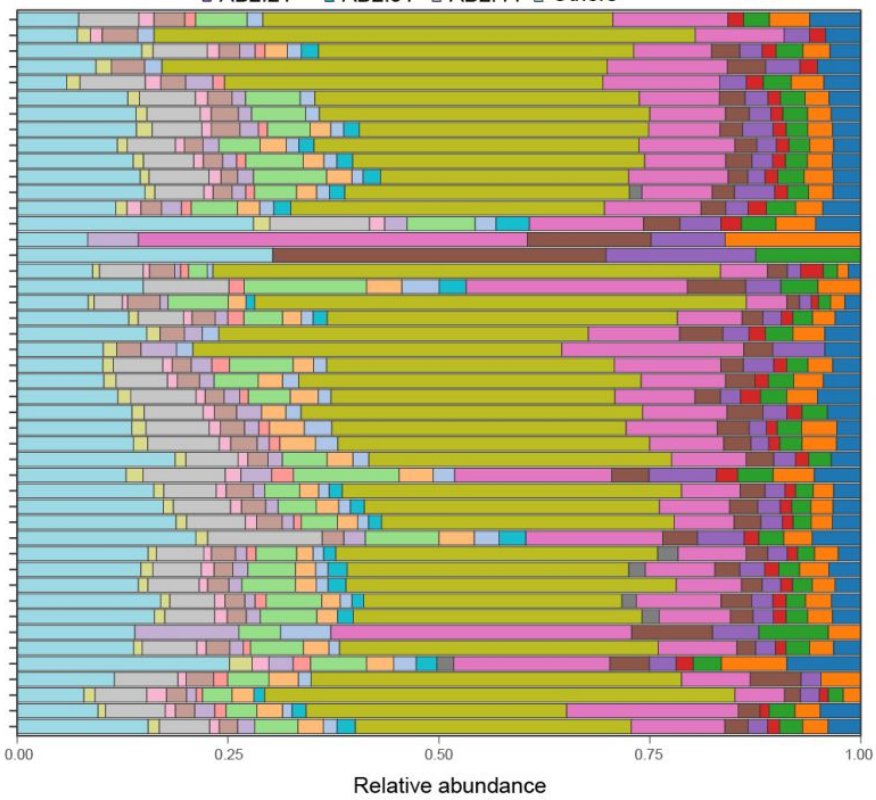

Figure S13 Microbial compositions of significantly different KEGG Modules. (a)

$\mathrm{AB}_{1}$ at phylum level. (b) $\mathrm{AB}_{1}$ at genome level. (c) $\mathrm{AB}_{2}$ at phylum level. (d) $\mathrm{AB}_{2}$ at

genome level. 


\section{References}

1. Krustok, I.; Odlare, M.; Truu, J.; Nehrenheim, E., Inhibition of nitrification in municipal wastewater-treating photobioreactors: Effect on algal growth and nutrient uptake. Bioresour. Technol. 2016, 202, 238-243.

2. Kujawinski, E. B.; Behn, M. D., Automated Analysis of Electrospray Ionization Fourier Transform Ion Cyclotron Resonance Mass Spectra of Natural Organic Matter. Anal. Chem. 2006, 78, (13), 4363-4373.

3. Kellerman, A. M.; Dittmar, T.; Kothawala, D. N.; Tranvik, L. J., Chemodiversity of dissolved organic matter in lakes driven by climate and hydrology. Nat. Commun. 2014, 5,8 .

4. D'Andrilli, J.; Cooper, W. T.; Foreman, C. M.; Marshall, A. G., An ultrahighresolution mass spectrometry index to estimate natural organic matter lability. Rapid Commun. Mass Spectrom. 2015, 29, (24), 2385-2401.

5. Zhang, B.; Shan, C.; Hao, Z.; Liu, J.; Wu, B.; Pan, B., Transformation of dissolved organic matter during full-scale treatment of integrated chemical wastewater: Molecular composition correlated with spectral indexes and acute toxicity. Water Res. 2019, 157, 472-482.

6. Callahan, B. J.; McMurdie, P. J.; Rosen, M. J.; Han, A. W.; Johnson, A. J. A.; Holmes, S. P., DADA2: High-resolution sample inference from Illumina amplicon data. Nat. Methods 2016, 13, (7), 581-583.

7. Bokulich, N. A.; Kaehler, B. D.; Rideout, J. R.; Dillon, M.; Bolyen, E.; Knight, R.; Huttley, G. A.; Gregory Caporaso, J., Optimizing taxonomic classification of marker-gene amplicon sequences with QIIME 2's q2-feature-classifier plugin. Microbiome 2018, 6, (1), 90.

8. McDonald, D.; Price, M. N.; Goodrich, J.; Nawrocki, E. P.; DeSantis, T. Z.; Probst, A.; Andersen, G. L.; Knight, R.; Hugenholtz, P., An improved Greengenes taxonomy with explicit ranks for ecological and evolutionary analyses of bacteria and archaea. ISME J. 2012, 6, (3), 610-618.

9. Janssen, S.; McDonald, D.; Gonzalez, A.; Navas-Molina, J. A.; Jiang, L.; Xu, Z. Z.; Winker, K.; Kado, D. M.; Orwoll, E.; Manary, M.; Mirarab, S.; Knight, R., Phylogenetic Placement of Exact Amplicon Sequences Improves Associations with Clinical Information. mSystems 2018, 3, (3), e00021-18.

10. Faith, D. P., Conservation evaluation and phylogenetic diversity. Biol. Conserv. 1992, 61, (1), 1-10.

11. Chao, A., Nonparametric-estimation of the number of classes in a population. Scandinavian Journal of Statistics 1984, 11, (4), 265-270.

12. Shannon, C. E., A mathematical theory of communication. Bell System Technical Journal 1948, 27, (4), 623-656.

13. Simpson, E. H., Measurement of diversity. Nature 1949, 163, (4148), 688-688.

14. Lozupone, C. A.; Hamady, M.; Kelley, S. T.; Knight, R., Quantitative and qualitative beta diversity measures lead to different insights into factors that structure microbial communities. Appl. Environ. Microbiol. 2007, 73, (5), 1576-1585. 
15. Bolyen, E.; Rideout, J. R.; Dillon, M. R.; Bokulich, N. A.; Abnet, C. C.; AlGhalith, G. A.; Alexander, H.; Alm, E. J.; Arumugam, M.; Asnicar, F.; Bai, Y.; Bisanz, J. E.; Bittinger, K.; Brejnrod, A.; Brislawn, C. J.; Brown, C. T.; Callahan, B. J.; Caraballo-Rodríguez, A. M.; Chase, J.; Cope, E. K.; Da Silva, R.; Diener, C.; Dorrestein, P. C.; Douglas, G. M.; Durall, D. M.; Duvallet, C.; Edwardson, C. F.; Ernst, M.; Estaki, M.; Fouquier, J.; Gauglitz, J. M.; Gibbons, S. M.; Gibson, D. L.; Gonzalez, A.; Gorlick, K.; Guo, J.; Hillmann, B.; Holmes, S.; Holste, H.; Huttenhower, C.; Huttley, G. A.; Janssen, S.; Jarmusch, A. K.; Jiang, L.; Kaehler, B. D.; Kang, K. B.; Keefe, C. R.; Keim, P.; Kelley, S. T.; Knights, D.; Koester, I.; Kosciolek, T.; Kreps, J.; Langille, M. G. I.; Lee, J.; Ley, R.; Liu, Y.-X.; Loftfield, E.; Lozupone, C.; Maher, M.; Marotz, C.; Martin, B. D.; McDonald, D.; McIver, L. J.; Melnik, A. V.; Metcalf, J. L.; Morgan, S. C.; Morton, J. T.; Naimey, A. T.; NavasMolina, J. A.; Nothias, L. F.; Orchanian, S. B.; Pearson, T.; Peoples, S. L.; Petras, D.; Preuss, M. L.; Pruesse, E.; Rasmussen, L. B.; Rivers, A.; Robeson, M. S.; Rosenthal, P.; Segata, N.; Shaffer, M.; Shiffer, A.; Sinha, R.; Song, S. J.; Spear, J. R.; Swafford, A. D.; Thompson, L. R.; Torres, P. J.; Trinh, P.; Tripathi, A.; Turnbaugh, P. J.; UlHasan, S.; van der Hooft, J. J. J.; Vargas, F.; Vázquez-Baeza, Y.; Vogtmann, E.; von Hippel, M.; Walters, W.; Wan, Y.; Wang, M.; Warren, J.; Weber, K. C.; Williamson, C. H. D.; Willis, A. D.; Xu, Z. Z.; Zaneveld, J. R.; Zhang, Y.; Zhu, Q.; Knight, R.; Caporaso, J. G., Reproducible, interactive, scalable and extensible microbiome data science using QIIME 2. Nat. Biotechnol. 2019, 37, (8), 852-857.

16. Brown, J.; Pirrung, M.; McCue, L. A., FQC Dashboard: integrates FastQC results into a web-based, interactive, and extensible FASTQ quality control tool.

Bioinformatics 2017, 33, (19), 3137-3139.

17. Nurk, S.; Meleshko, D.; Korobeynikov, A.; Pevzner, P. A., metaSPAdes: a new versatile metagenomic assembler. Genome Res. 2017, 27, (5), 824-834.

18. Alneberg, J.; Bjarnason, B. S.; de Bruijn, I.; Schirmer, M.; Quick, J.; Ijaz, U. Z.; Lahti, L.; Loman, N. J.; Andersson, A. F.; Quince, C., Binning metagenomic contigs by coverage and composition. Nat. Methods 2014, 11, 1144.

19. Kang, D. D.; Li, F.; Kirton, E.; Thomas, A.; Egan, R.; An, H.; Wang, Z., MetaBAT 2: an adaptive binning algorithm for robust and efficient genome reconstruction from metagenome assemblies. PeerJ 2019, 7, e7359.

20. Wu, Y.-W.; Tang, Y.-H.; Tringe, S. G.; Simmons, B. A.; Singer, S. W., MaxBin: an automated binning method to recover individual genomes from metagenomes using an expectation-maximization algorithm. Microbiome 2014, 2, (1), 26.

21. Uritskiy, G. V.; DiRuggiero, J.; Taylor, J. J. M., MetaWRAP—a flexible pipeline for genome-resolved metagenomic data analysis. Microbiome 2018, 6, (1), 158.

22. Faust, K.; Raes, J., CoNet app: inference of biological association networks using Cytoscape. F1000Res 2016, 5, 1519. 